Tôhoku Math. Journ.

39 (1987), 519-532.

\title{
CERTAIN COMPACT COMPLEX MANIFOLDS WITH INFINITE CYCLIC FUNDAMENTAL GROUPS
}

\author{
HIROYASU TSUCHIHASHI
}

(Received August 19, 1986)

Introduction. In this paper, we give a method of constructing certain examples of compact complex manifolds $U$ with $\pi_{1}(U) \simeq Z$ and study the structure of $U$. Those manifolds are toroidal compactifications of the quotient spaces of open sets of algebraic tori $\left(C^{\times}\right)^{r}$ by the groups $g^{z}$ generated by elements $g$ in $G L(r, Z)$ satisfying certain conditions (Definition 1.1). As examples of such $g$, we can take integral matrices whose entries are all positive. Since the first Betti numbers of such manifolds are equal to one, they are not Kähler manifolds. In the two-dimensional case, those manifolds $U$ are hyperbolic Inoue surfaces or half Inoue surfaces (see [3]). Hence we may regard our examples as higher-dimensional analogues of hyperbolic Inoue surfaces. On the other hand, one of them is bimeromorphic to that constructed by Kato [6]. Therefore, we call them Inoue-Kato manifolds. (The name was suggested by Ishida.) Sankaran [9] also constructs certain examples of compact complex manifolds $M$, which are in another sense higher-dimensional analogues of hyperbolic Inoue surfaces, and whose fundamental groups are free abelian groups of rank $\operatorname{dim} M-1$.

This paper is organized as follows. In Section 1 and Section 2, we construct compact complex manifolds mentioned above and their degenerations, respectively. In Section 3, we show that a part of them contain global spherical shells. In Section 4 and Section 5, we calculate some of their analytic invariants. We show some examples in Section 6 .

The author would like to thank Professor T. Oda who pointed out the fact in Proposition 1.4.

1. The construction. Let $N \simeq Z^{r}$ be a free $Z$-module of rank $r$ and let $T=N \otimes C^{\times}$be an algebraic torus of rank $r$.

DEFINITION 1.1. Let $K(N)$ be the set of $Z$-linear transformations $g$ of $N$ satisfying the following condition.

$g$ has a simple real eigenvalue $\lambda=\lambda(g)$ such that $|\eta|<\lambda$ for all the

Partly supported by the Grants-in-Aid for Encouragement of Young Scientists, The Ministry of Education, Science and Culture, Japan. 
other eigenvalues $\eta$ of $g$.

Clearly, we have:

Proposition 1.2. If $g$ is in $K(N)$, then ${ }^{t} g$ is in $K\left(N^{*}\right)$, where the transpose ${ }^{t} g$ is the linear transformation of $N^{*}:=\operatorname{Hom}(N, \boldsymbol{Z})$ definined by $\langle m, g n\rangle=\left\langle{ }^{t} g m, n\right\rangle$, for all $m \in N^{*}$ and for all $n \in N$.

Proposition 1.3. Let $g$ be in $K(N)$. Then there exists an open convex cone $C$ such that the closure of $g C$ is contained in $C \cup\{0\}$, that $H(g):=$ $\cup_{l \in Z} g^{l} C$ is a half-space of $N_{R}$ and that $L(g):=\cap_{l \in Z} g^{l} C$ is a half-line of $N_{R}$, where we denote by the same letter $g$, the image of $g$ under the natural map $G L(N) \rightarrow G L\left(N_{R}\right)$.

Proof. Let $v$ and $v^{*}$ be eigenvectors of $g$ and ${ }^{t} g$, respectively, associated with the real eigenvalue $\lambda(g)$. Then clearly, $\left\langle v^{*}, v\right\rangle \neq 0$. Hence we may assume that $\left\langle v^{*}, v\right\rangle>0$. Then the half-space $H:=\left\{y \in N_{R}\left|\left\langle v^{*}, y\right\rangle\right\rangle\right.$ 0\} contains the half-line $L:=\boldsymbol{R}_{>0} v$. Take an open polygonal cone $C_{0}=$ $\boldsymbol{R}_{>0} n_{1}+\boldsymbol{R}_{>0} n_{2}+\cdots+\boldsymbol{R}_{>0} n_{s}$ containing $L$ and contained in $H$. Then $\cap_{l \in Z} g^{l} C_{0}=L$. Hence there exists a positive integer $l_{0}$ such that the closure of $g^{l_{0}} C_{0}$ is contained in $C_{0} \cup\{0\}$. Let $C_{j}=\boldsymbol{R}_{>0} n_{1}(j)+\boldsymbol{R}_{>0} n_{2}(j)+\cdots+$ $\boldsymbol{R}_{>0} n_{s}(j)$, where $n_{k}(j)=n_{k}+\left(j \varepsilon / l_{0}\right) v$. Then the closure of $g^{l_{0}} C_{0}$ is contained also in $C_{l_{0}} \cup\{0\}$ for a positive real number $\varepsilon$ small enough. Moreover, the closure of $C_{j+1}$ is contained in $C_{j} \cup\{0\}$. Let $C=C_{l_{0-1}} \cap g C_{l_{0-2}} \cap \cdots \cap$ $g^{l_{0}-1} C_{0}$. Then the closure of $g C=g^{l_{0}} C_{0} \cap g C_{l_{0-1}} \cap \cdots \cap g^{l_{0}-1} C_{1}$ is contained in $C \cup\{0\}$. Since also $C$ contains $L$ and contained in $H$, we have $\cap_{l \in Z} g^{l} C=L$ and $\cup_{l \in Z} g^{l} C=H$.

q.e.d.

Let $g$ be in $K(N)$. Then we see by the above proposition that the cyclic group $g^{\boldsymbol{Z}}$ generated by $g$ acts on $D(g):=(H(g) \backslash L(g)) / \boldsymbol{R}_{>0}$ properly discontinuously and without fixed points and that the quotient $D(g) / g^{\mathbf{Z}}$ is compact. Moreover, $D(g) / g^{Z}$ is homeomorphic to $S^{r-2} \times S^{1}$, if $g$ is in $S L(N)$.

Proposition 1.4. Let $g$ be in the group $G L(N)$ of $\boldsymbol{Z}$-automorphisms of $N$ and let $C$ be the interior of a non-singular rational cone of dimension $r$ in $N_{R}$. Assume that $g C$ is contained in $C$ and that the closure of $g^{l} C$ is contained in $C \cup\{0\}$, for a positive integer $l$. Then $g$ is in $K(N)$.

Proof. By assumption, there exists a $Z$-basis $\left\{n_{1}, n_{2}, \cdots, n_{r}\right\}$ of $N$ with $C=\boldsymbol{R}_{>0} n_{1}+\boldsymbol{R}_{>0} n_{2}+\cdots+\boldsymbol{R}_{>0} n_{r}$. Then $g$ (resp. $g^{l}$ ) is represented with respect to the basis $\left\{n_{1}, n_{2}, \cdots, n_{r}\right\}$ by a matrix whose entries are all non-negative (resp. positive) integers. Hence by the Perron-Frobenius theorem (see [10, Ex. 37]), $g$ (resp. $g^{l}$ ) has a real eigenvalue $\lambda$ (resp. a 
simple real eigenvalue $\lambda^{\prime}$ ) such that $\lambda \geqq|\eta|$ (resp. $\left.\left.\lambda^{\prime}\right\rangle\left|\eta^{\prime}\right|\right)$ for the other eigenvalues $\eta$ of $g$ (resp. $\eta^{\prime}$ of $\left.g^{l}\right)$. Here clearly, $\lambda^{\prime}=\lambda^{l}$. Therefore, $\lambda$ is a simple real eigenvalue of $g$ and $\lambda>|\eta|$ for the other eigenvalues $\eta$ of $g$.

q.e.d.

Proposition 1.5. Let $g$ be in $K(N)$. Then $g^{z}$ acts on $H(g)$ properly discontinuously and without fixed points.

Proof. Let $v$ and $v^{*}$ be the same as in the proof of Proposition 1.3. By Proposition 1.3, we have a cone $C$ such that the closure of $g C$ is contained in $C \cup\{0\}$. Let $F=\left\{y \in C \mid\left\langle v^{*}, y\right\rangle>1\right\}$. Then $\cup_{l \in Z} g^{l} F=H(g)$, $\cap_{l \in Z} g^{l} F=\varnothing$ and the closure of $g F$ is contained in $F$, because $\left\langle v^{*}, g y\right\rangle=$ $\left\langle{ }^{t} g v^{*}, y\right\rangle=\lambda(g)\left\langle v^{*}, y\right\rangle>\lambda(g)>1$ for any $y$ in $F$. Hence the action of $g^{\mathbf{Z}}$ on $H(g)$ is properly discontinuous and fixed point free. q.e.d.

In the following, we use the notation in [8]. Let $g$ be in $K(N)$ and let $\widetilde{W}=\operatorname{ord}^{-1}(H(g))$ be the inverse image of $H(g)$ under the $G L(N)$ equivariant map ord $=-\log ||: T \rightarrow N_{R}$. Then the quotient $W:=\widetilde{W} / g^{\mathbf{Z}}$ of $\widetilde{W}$ with respect to the action of $g^{z}$ is a complex manifold by the above proposition. In the following, we construct a toroidal compactification of $W$. First, we show that there exists a $g^{z}$-invariant r.p.p. decomposition $\Sigma$ in $N$ with $|\Sigma|\left(:=\cup_{\sigma \in \Sigma} \sigma\right)=(H(g) \backslash L(g)) \cup\{0\}$. We can take a strongly convex rational polyhedral cone $C$ such that $g C$ is contained in $\operatorname{Int}(C) \cup\{0\}$, that $\cup_{l \in Z} g^{l} C=H(g) \cup\{0\}$ and that $\cap_{l \in Z} g^{l} C=L(g) \cup\{0\}$, by Proposition 1.3. Let $\Lambda=\{$ faces of $C\} \backslash\{C\}$. Then since $\Lambda \cup g \Lambda$ is an r.p.p. decomposition in $N$, we have a complete r.p.p. decomposition $\Lambda^{\prime}$ containing $\Lambda \cup g \Lambda$, by [11, Theorem 3] and [8, Theorem 4.1]. Let $\Sigma_{0}=\left\{\sigma \in \Lambda^{\prime} \mid \sigma \subset C \backslash \operatorname{Int}(g C)\right\}$. Then $\left|\Sigma_{0}\right|=C \backslash \operatorname{Int}(g C)$, because $C \backslash \operatorname{Int}(g C)$ is the closure of a connected component of $N_{R} \backslash|\Lambda \cup g \Lambda|$. Hence $\Sigma=\left\{g^{l} \sigma \mid \sigma \in \Sigma_{0}, l \in Z\right\}$ is a $g^{z}$-invariant r.p.p. decomposition in $N$ and $|\Sigma|=\cup_{l \in Z}\left(g^{l} C \backslash \operatorname{Int}\left(g^{l+1} C\right)\right)=\left(\cup_{l \in Z} g^{l} C\right) \backslash$ $\left(\cap_{l \in Z} \operatorname{Int}\left(g^{l+1} C\right)\right)=(H(g) \backslash L(g)) \cup\{0\}$. Let $\widetilde{X}=T \operatorname{emb}(\Sigma) \backslash T$ and let $\widetilde{U}=$ $\widetilde{W} \cup \widetilde{X}$. Then $\widetilde{U}$ is an open set of $T \operatorname{emb}(\Sigma)$ and is invariant under the action of $g^{z}$.

Proposition 1.6. $\widetilde{U}$ is simply connected.

Proof. Note that the inclusion map $\widetilde{W} \hookrightarrow T$ induces an isomorphism $\pi_{1}(\tilde{W}) \simeq \pi_{1}(T)$ of the fundamental groups. Hence we get the assertion of the proposition in the same way as in the proof of [8, Proposition 10.2].

q.e.d.

We obtain from $\Sigma$, a $g^{z}$-invariant polygonal decomposition $\Delta:=$ $\left\{(\sigma \backslash\{0\}) / \boldsymbol{R}_{>0} \mid \sigma \in \Sigma \backslash\{\{0\}\}\right\}$ on $D(g)$, which coincides with the dual graph of $\widetilde{X}$. Since $g^{z}$ has no fixed points on $D(g)$, neither does it on $\widetilde{X}$. Let 
$U=\tilde{U} / g^{z}$ and let $X=\tilde{X} / g^{z}$. Then $X$ is a divisor on $U$ and the dual graph of $X$ is the graph on $D(g) / g^{z}$ which is the image of $\Delta$ under the projection $D(g) \rightarrow D(g) / g^{Z}$.

PRoposition 1.7. $U$ is an $r$-dimensional compact complex variety with the fundamental group $\pi_{1}(U) \simeq \boldsymbol{Z}$.

Proof. Since $g^{z}$ has no fixed points on $\tilde{X}$ and on $\tilde{W}$, neither does it on $\widetilde{U}=\tilde{W} \cup \tilde{X}$. Let $F$ be the same as in the proof of Proposition 1.5. Then the closure $G$ of $F \backslash g F$ in $\tilde{U} / C T$ is compact, where $C T$ is the compact real torus $N \otimes U(1)$ in $T$. Hence the inverse image of $G$ under the map ord: $\widetilde{U} \rightarrow \widetilde{U} / C T$ is also compact and is a fundamental domain with respect to the action of $g^{z}$. Therefore, $U$ is an $r$-dimensional compact complex variety. Moreover, $\pi_{1}(U) \simeq g^{Z} \simeq Z$ by Proposition 1.6. q.e.d.

Assume that $\Sigma$ consists of non-singular cones. Then $\widetilde{U}$ and $U$ are complex manifolds. Moreover, the dual graph $\Delta$ of $\tilde{X}$ is a triangulation.

REMARK. When $r=2, U$ is a hyperbolic Inoue surface or is a half Inoue surface, according as $g$ belongs to $S L(N)$ or not.

2. Degenerations. Since $\lambda$ in Definition 1.1 is greater than one, we have:

Proposition 2.1. If $g$ is in $K(N)$, then $\widehat{g}_{ \pm}$is in $K(N \oplus \boldsymbol{Z})$ where $\hat{\boldsymbol{g}}_{ \pm}$is the linear transformation of $N \oplus \boldsymbol{Z}$ sending $(n, l)$ to $(g n, \pm l)$.

Let $g$ be in $K(N)$ and assume that there exists an r.p.p. decomposition $\Sigma$ with $|\Sigma|=(H(g) \backslash L(g)) \cup\{0\}$. Let $\Lambda=\left\{\boldsymbol{R}_{\geqq 0} 1,\{0\}, \boldsymbol{R}_{\geq 0}(-1)\right\}$. Then $\Lambda$ is an r.p.p. decomposition in $\boldsymbol{Z}$ and $B:=T_{Z} \mathrm{emb}(\Lambda)$ is a non-singular rational curve. Assume that there exists an r.p.p. decomposition $\hat{\Sigma}$ in $N \oplus \boldsymbol{Z}$ satisfying the following condition.

(D) $\hat{\Sigma}$ is $\left(\hat{g}_{+}\right)^{z}$-invariant, $\left.|\hat{\Sigma}|=((H(g) \times \boldsymbol{R}) \backslash(L(g)) \times\{0\})\right) \cup\{0\}$, the subcomplex $\left\{\sigma \in \hat{\Sigma} \mid \sigma \subset N_{R}\right\}$ of $\hat{\Sigma}$ is equal to $\Sigma$ and the natural projection $N \oplus \boldsymbol{Z} \rightarrow \boldsymbol{Z}$ induces a morphism $(N \oplus \boldsymbol{Z}, \hat{\Sigma}) \rightarrow(\boldsymbol{Z}, \Lambda)$ of r.p.p. decompositions.

Then we have an $(r+1)$-dimensional compact complex variety $\mathscr{U}:=$ $\left(\operatorname{ord}^{-1}(H(g) \times \boldsymbol{R}) \cup\left(T_{N \oplus \boldsymbol{Z}} \mathrm{emb}(\hat{\Sigma}) \backslash T_{N \oplus \boldsymbol{Z}}\right)\right) /\left(\hat{g}_{+}\right)^{\boldsymbol{Z}}$, a divisor $\mathscr{Z}:=\left(T_{N \oplus \boldsymbol{Z}} \mathrm{emb}(\hat{\Sigma}) \backslash\right.$ $\left.T_{N \oplus \mathbb{Z}}\right) /\left(\widehat{g}_{+}\right)^{Z}$ on $\mathscr{U}$ and a holomorphic map $\varphi: \mathscr{U} \rightarrow B$ with $\varphi^{-1}\left(T_{Z}\right) \simeq$ $U \times T_{z}\left(\varphi^{-1}\left(T_{z}\right) \cap \mathscr{Z} \simeq X \times T_{Z}\right)$

THEOREM 2.2. Assume that there exist a convex rational cone $C$ and $a$ set $\Sigma_{r}^{0}$ of $r$-dimensional cones in $\Sigma$ such that $g C \subset \operatorname{Int}(C) \cup\{0\}$ and that $\left|\Sigma_{r}^{0}\right|=C \backslash \operatorname{Int}(g C)$. Then there exists an r.p.p. decomposition $\hat{\Sigma}$ in $N \oplus \boldsymbol{Z}$ satisfying the above condition (D). Moreover, $\varphi^{-1}\left(\operatorname{orb}\left(\boldsymbol{R}_{\geq 0} 1\right)\right) \simeq$ 
$\varphi^{-1}\left(\operatorname{orb}\left(\boldsymbol{R}_{\geq 0}(-1)\right)\right)$ is a toric variety intersecting itself along two disjoint divisors.

Proof. First, we note that $\Sigma_{r}^{0}$ consists of representatives of $r$ dimensional cones of $\Sigma$ modulo $g^{Z}$, i.e., $\left\{g^{l} \sigma \mid l \in Z, \sigma \in \Sigma_{r}^{0}\right\}=\{r$-dimensional cones in $\Sigma$ \} and $l=0$ if $g^{l} \sigma=\tau$ for $\sigma, \tau \in \Sigma_{r}^{0}$. Take an element $v$ in $N$ so that $g v-v$ is contained in $\operatorname{Int}(g C)$ and let $\hat{C}=\left(\boldsymbol{R}_{\geqq 0}(v, 1)+C\right) \cup$ $\left(\boldsymbol{R}_{\geqq 0}(v,-1)+C\right)$. Then $\widehat{g}_{+} \hat{C}$ is contained in $\operatorname{Int}(\hat{C}) \cup\{0\}$. Let $\Sigma^{0}=$ faces of $\left.\sigma \mid \sigma \in \Sigma_{r}^{0}\right\}$ and let $\hat{\Sigma}^{0}=\left\{\hat{\sigma}_{ \pm}, \widetilde{\tau}_{ \pm} \mid \sigma, \tau \in \Sigma^{0}, \tau \subset g C\right\}$, where $\hat{\sigma}_{ \pm}=\boldsymbol{R}_{\geqq 0}(v, \pm 1)+\sigma$ and $\tilde{\tau}_{ \pm}=\boldsymbol{R}_{\geqq 0}(v, \pm 1)+\boldsymbol{R}_{\geqq 0}(g v, \pm 1)+\tau$. Then we can verify that $g^{l} \eta \cap \lambda$ are faces of $g^{l} \eta$ and $\lambda$, for $\eta, \lambda \in \hat{\Sigma}^{0}$ and for $l \in Z$ and that $\left|\hat{\Sigma}^{0}\right|=\widehat{C} \backslash \operatorname{Int}\left(\hat{g}_{+} \hat{C}\right)$. Hence $\hat{\Sigma}:=\left\{\right.$ faces of $\left.\left(\hat{g}_{+}\right)^{l} \lambda \mid \lambda \in \hat{\Sigma}^{0}, l \in \boldsymbol{Z}\right\}$ is an r.p.p. decomposition in $N \oplus \boldsymbol{Z}$ and satisfies the condition (D). The last assertion follows from the construction of $\hat{\Sigma}$.

q.e.d.

COROLlary 2.3. Let $g$ be in $K(N)$ and assume that there exist an r.p.p. decomposition $\Sigma$ with $|\Sigma|=(H(g) \backslash L(g)) \cup\{0\}$, a positive integer $l$, a convex rational cone $C$ and a set $\Sigma_{r}^{0}$ of $r$-dimensional cones in $\Sigma$ such that $\left|\Sigma_{r}^{0}\right|=C \backslash \operatorname{Int}\left(g^{l} C\right)$ and that $g^{l} C \subset \operatorname{Int}(C) \cup\{0\}$. Then there exists an r.p.p. decomposition $\hat{\Sigma}$ satisfying the condition (D).

Proof. By Theorem 2.2, we have a $\left(\hat{g}_{+}\right)^{l z}$-invariant r.p.p. decomposition $\hat{\Xi}$ such that $|\hat{\Xi}|=((H(g) \times \boldsymbol{R}) \backslash(L(g) \times\{0\})) \cup\{0\}$ and that $\left\{\sigma \in \hat{\Xi} \mid \sigma \subset N_{R}\right\}=$ $\Sigma$. Let $\hat{\Sigma}=\left\{h_{1} \sigma_{1} \cap h_{2} \sigma_{2} \cap \cdots \cap h_{l} \sigma_{l} \mid \sigma_{i} \in \hat{\Xi}\right\}$, where $h_{i}=\left(\hat{g}_{+}\right)^{i}$. Then $\hat{\Sigma}$ is $\left(\hat{g}_{+}\right)^{z}$-invariant, consists of rational cones, $|\hat{\Sigma}|=|\hat{\Xi}|$ and $\left\{\sigma \in \hat{\Sigma} \mid \sigma \subset N_{R}\right\}=\Sigma$, because $\Sigma$ is $g^{z}$-invariant. Hence it is sufficient to show that $\hat{\Sigma}$ is an r.p.p. decomposition. Let $\tau$ be a face of an element $\sigma=h_{1} \sigma_{1} \cap h_{\mathrm{z}} \sigma_{2} \cap \cdots \cap$ $h_{l} \sigma_{l}$ in $\hat{\Sigma}$. Then $\tau=\sigma \cap x^{\perp}$, for an element $x=x_{1}+x_{2}+\cdots+x_{l}$ in $\sigma^{\vee}=\left(h_{1} \sigma_{1}\right)^{\vee}+\left(h_{2} \sigma_{2}\right)^{\vee}+\cdots+\left(h_{l} \sigma_{l}\right)^{\vee}\left(x_{i} \in\left(h_{i} \sigma_{i}\right)^{\vee}\right)$, where $\sigma^{\vee}$ is the dual cone of $\sigma$ and $x^{\perp}=\left\{y \in N_{R} \mid\langle x, y\rangle=0\right\}$. Let $\tau_{i}=h_{i} \sigma_{i} \cap x_{i}^{\perp}$. Then $\tau_{i}$ is a face of $h_{i} \sigma_{i}$ and

$$
\begin{aligned}
\tau & =\{y \in \sigma \mid\langle x, y\rangle=0\} \\
& =\left\{y \in \sigma \mid\left\langle x_{1}, y\right\rangle=\left\langle x_{2}, y\right\rangle=\cdots=\left\langle x_{l}, y\right\rangle=0\right\}=\tau_{1} \cap \tau_{2} \cap \cdots \cap \tau_{l} \in \hat{\Sigma},
\end{aligned}
$$

because $\left\langle x_{i}, y\right\rangle \geqq 0$ for $y \in \sigma$. Next, let $\sigma=h_{1} \sigma_{1} \cap h_{2} \sigma_{2} \cap \cdots \cap h_{l} \sigma_{l}$ and $\tau=$ $h_{1} \tau_{1} \cap h_{2} \tau_{2} \cap \cdots \cap h_{l} \tau_{l}$ be in $\hat{\Sigma}$. Then $\sigma \cap \tau=h_{1}\left(\sigma_{1} \cap \tau_{1}\right) \cap h_{2}\left(\sigma_{2} \cap \tau_{2}\right) \cap \cdots \cap$ $h_{l}\left(\sigma_{l} \cap \tau_{l}\right)$. Since $h_{i}\left(\sigma_{i} \cap \tau_{i}\right)$ is a face of $h_{i} \sigma_{i}$, there exists an element $x_{i}$ in $\left(h_{i} \sigma_{i}\right)^{\vee}$ with $h_{i}\left(\sigma_{i} \cap \tau_{i}\right)=h_{i} \sigma_{i} \cap x_{i}^{\perp}$. Hence

$$
\begin{aligned}
\sigma \cap \tau & =\left\{y \in \sigma \mid\left\langle x_{1}, y\right\rangle=\left\langle x_{2}, y\right\rangle=\cdots=\left\langle x_{l}, y\right\rangle=0\right\} \\
& =\left\{y \in \sigma \mid\left\langle x_{1}+x_{2}+\cdots+x_{l}, y\right\rangle=0\right\}
\end{aligned}
$$

is a face of $\sigma$, because $x_{1}+x_{2}+\cdots+x_{l} \in\left(h_{1} \sigma_{1}\right)^{\vee}+\left(h_{2} \sigma_{2}\right)^{\vee}+\cdots+\left(h_{l} \sigma_{l}\right)^{\vee}=$ $\sigma^{\vee}$.

q.e.d. 
3. Global spherical shells. We keep the notation in Section 1.

DEFinition 3.1. An open set $S$ of a complex manifold $U$ is a global spherical shell, if $U \backslash S$ is connected and if $S$ is biholomorphic to $\left\{\left.\left(z_{1}, z_{2}, \cdots, z_{r}\right) \in C^{r}\left|\alpha<\sum_{k=1}^{r}\right| z_{k}\right|^{2}<\beta\right\}$ for positive real numbers $\alpha$ and $\beta$ with $0<\alpha<\beta$.

See [5], for the properties of compact complex manifolds containing global spherical shells.

THEOREM 3.2. If there exists an $r$-dimensional non-singular rational cone $\sigma$ in $N_{R}$ such that $g \sigma \backslash\{0\}$ is contained in the interior of $\sigma$ and that $[\sigma]:=\{$ faces of $\sigma\} \backslash\{\sigma\}$ is contained in $\Sigma$, then $U$ contains a global spherical shell.

Proof. Let $\left\{n_{1}, n_{2}, \cdots, n_{r}\right\}$ be a $\boldsymbol{Z}$-basis of $N$ with $\boldsymbol{\sigma}=\boldsymbol{R}_{\geq 0} n_{1}+$ $\boldsymbol{R}_{\geqq 0} n_{2}+\cdots+\boldsymbol{R}_{\geqq 0} n_{r}$ and let $\left\{m_{1}, m_{2}, \cdots, m_{r}\right\}$ be the $\boldsymbol{Z}$-basis of $N^{*}$ dual to $\left\{n_{1}, n_{2}, \cdots, n_{r}\right\}$. Let $z_{i}$ be the holomorphic function on $T \operatorname{emb}([\sigma])$ $\left(\simeq C^{r} \backslash\{0\}\right)$ which is the natural extension of the character $m_{i} \otimes 1_{C^{\times}}: T \rightarrow$ $C^{\times}$of $m_{i}$. Then $\left(z_{1}, z_{2}, \cdots, z_{r}\right)$ is a global coordinate on $T \operatorname{emb}([\sigma])$. Let $S=\left\{\left.\left(z_{1}, z_{2}, \cdots, z_{r}\right) \in T \operatorname{emb}([\sigma])\left|\gamma-\varepsilon<\sum_{k=1}^{r}\right| z_{k}\right|^{2}<\gamma+\varepsilon\right\}$ for positive real numbers $\gamma$ and $\varepsilon$ with $\gamma-\varepsilon>0$ and $\gamma+\varepsilon<1$. Then we easily see that the image $\operatorname{ord}(S \backslash \tilde{X})=\left\{u_{1} n_{1}+u_{2} n_{2}+\cdots+u_{r} n_{r} \mid \gamma-\varepsilon<\exp \left(-2 u_{1}\right)+\right.$ $\left.\exp \left(-2 u_{2}\right)+\cdots+\exp \left(-2 u_{r}\right)<\gamma+\varepsilon\right\}$ of $S \backslash \tilde{X}$ under the map ord: $T \rightarrow N_{R}$ is contained in $\sigma \backslash\{0\} \subset H(g)$. Hence $S$ is contained in $\widetilde{U}$. Let $P_{ \pm}=$ $\left\{u_{1} n_{1}+u_{2} n_{2}+\cdots+u_{r} n_{r} \mid \exp \left(-2 u_{1}\right)+\exp \left(-2 u_{2}\right)+\cdots+\exp \left(-2 u_{r}\right)<\gamma \pm \varepsilon\right\}$. Then the closure of $g P_{+}$is contained in $P_{-}$for small enough $\varepsilon$, because $g n_{j}=a_{1 j} n_{1}+a_{2 j} n_{2}+\cdots+a_{r j} n_{r}$ with $a_{i j} \geqq 1$, for $j=1$ through $r$. Since $S \backslash \tilde{X}=\operatorname{ord}^{-1}\left(P_{+} \backslash \bar{P}_{-}\right)$and since $S \cap \tilde{X} \subset T \operatorname{emb}([\sigma]) \backslash T$, the restriction to $S$ of the quotient map $q: \widetilde{U} \rightarrow U$ is injective. Moreover, the image $q(S)$ of $S$ is global, i.e., $U \backslash q(S)$ is connected, because $U \backslash(q(S) \cup X)$ is the image under $q$ of the connected set $\operatorname{ord}^{-1}\left(\bar{P}_{-} \backslash g P_{+}\right)$.

q.e.d.

4. Invariants. We keep the notation in Section 1. Throughout this section, we assume that there exists an r.p.p. decomposition $\Sigma$ satisfying the conditions of Corollary 2.3 and consisting of non-singular cones. Let $\Theta_{\tilde{U}}(-\log \tilde{X})$ and $\Theta_{U}(-\log X)$ be the logarithmic tangent sheaves of $(\tilde{U}, \tilde{X})$ and $(U, X)$, respectively, and let $\Omega_{\widetilde{U}}^{1}(\log \widetilde{X})$ and $\Omega_{U}^{1}(\log X)$ be the dual sheaves of $\Theta_{\tilde{U}}(-\log \tilde{X})$ and $\Theta_{U}(-\log X)$, respectively. The first purpose of this section is to prove the following proposition.

Proposition 4.1.

$$
H^{i}\left(U, \mathcal{O}_{U}\right) \simeq\left\{\begin{array}{lll}
C & \text { for } & i=0,1 \\
0 & \text { for } & i \geqq 2,
\end{array}\right.
$$


and

$$
H^{i}\left(U, \Theta_{U}(-\log X)\right) \simeq \begin{cases}\operatorname{ker}(g-1) & \text { for } i=0 \\ \operatorname{coker}(g-1) & \text { for } i=1 \\ 0 & \text { for } i \geqq 2\end{cases}
$$

$$
H^{i}\left(U, \Omega_{U}^{1}(\log X)\right) \simeq \begin{cases}\operatorname{ker}\left({ }^{t} g-1\right) & \text { for } i=0 \\ \operatorname{coker}\left({ }^{t} g-1\right) & \text { for } i=1 \\ 0 & \text { for } i \geqq 2,\end{cases}
$$

where $(g-1): N_{c} \rightarrow N_{c}$ and $\left({ }^{t} g-1\right): N_{c}^{*} \rightarrow N_{c}^{*}$ are the $C$-linear maps sending $l$ and $l^{*}$ to $g l-l$ and ${ }^{t} g l^{*}-l^{*}$, respectively.

For the proof, we need some lemmas. Let $\mathscr{F}=q_{*}^{g Z} \tilde{\mathscr{F}}$, for a locally free sheaf $\tilde{\mathscr{F}}$ on $\widetilde{U}$ with an action of $g^{z}$, where $g: \widetilde{U} \rightarrow U=\widetilde{U} / g^{z}$ is the quotient map and $q_{*}^{g Z} \mathscr{F}^{\sim}$ denotes the subsheaf of $q_{*} \tilde{\mathscr{F}}$ consisting of germs of $g^{z}$-invariant sections. Then by [2, Corollary 3 to Theorem 5.3.1], we have the spectral sequence:

$$
E_{2}^{p, q}\left(g^{z}, \tilde{\mathscr{F}}\right)=H^{p}\left(g^{z}, H^{q}(\widetilde{U}, \tilde{\mathscr{F}})\right) \Rightarrow H^{p+q}(U, \mathscr{F}) .
$$

Here we note that $\mathscr{F}=\mathcal{O}_{U}, \Omega_{U}^{1}(\log X)$ or $\Theta_{U}(-\log X)$, according as $\tilde{\mathscr{F}}=$ $\sigma_{\tilde{U}}, \Omega_{\widetilde{U}}^{1}(\log \tilde{X})$ or $\Theta_{\widetilde{U}}(-\log \widetilde{X})$. Since $g^{Z}$ is a free group, we have $E_{2}^{p, q}\left(g^{z}, \tilde{\mathscr{F}}^{\prime}\right)=0$ for $p>1$. Hence the above spectral sequence degenerates and $H^{q}(U, \mathscr{F}) \simeq E_{2}^{0, q}\left(g^{z}, \tilde{F}^{2}\right) \oplus E_{2}^{1, q-1}\left(g^{z}, \tilde{F}\right)$. First, we calculate $E_{2}^{p, 0},\left(g^{z}, \tilde{\mathscr{F}}\right)$ for $p=0,1$ and for $\tilde{\mathscr{F}}=\mathcal{O}_{\tilde{U}}, \Theta_{\tilde{U}}(-\log \tilde{X}), \Omega_{\widetilde{U}}^{1}(\log \tilde{X})$.

LEMMA 4.2. $H^{0}\left(\widetilde{U}, \mathscr{O}_{\widetilde{U}}\right) \simeq \boldsymbol{C}$.

Proof. Since $\widetilde{U}$ is an open set of $T \operatorname{emb}(\Sigma)$, any holomorphic function $f$ on $\widetilde{U}$ is expressed as a series

$$
f=\sum_{m \in N^{*}} c_{m} e(m)
$$

where $\boldsymbol{e}(m)$ is the natural extension to $T \operatorname{emb}(\Sigma)$ of the character $m \otimes 1_{c \times}: T \rightarrow C^{\times}$of $m$. Here $c_{m}$ must vanish, if $\langle m, n\rangle<0$ for a nonzero element $n \in N$ with $\boldsymbol{R}_{\geq 0} n \in \Sigma$, because $\boldsymbol{e}(m)$ has poles along $\operatorname{orb}\left(\boldsymbol{R}_{\geqq 0} n\right) \subset \tilde{U}$. However,

$$
\begin{aligned}
\left\{m \in N^{*} \mid\langle m, n\rangle \geqq 0 \text { for all } n \in N \text { with } \boldsymbol{R}_{\geqq 0} n \in \Sigma\right\} \\
\quad=\left\{m \in N^{*} \mid\langle m, y\rangle \geqq 0 \text { for all } y \text { in } H(g)\right\} \\
=\left(L\left({ }^{t} g\right) \cup\{0\}\right) \cap N^{*}=\{0\},
\end{aligned}
$$

because $|\Sigma|=(H(g) \backslash L(g)) \cup\{0\}$. Hence $f=c_{0}$ is a constant function. Therefore, $H^{0}\left(\widetilde{U}, \mathscr{O}_{\widetilde{U}}\right) \simeq \boldsymbol{C}$.

q.e.d.

By [4, Proposition 1.12], there are $g^{z}$-equivariant isomorphisms 
$\Theta_{\tilde{U}}(-\log \tilde{X}) \simeq \mathcal{O}_{\tilde{U}} \otimes_{\mathbf{z}} N$ and $\Omega_{\widetilde{U}}^{1}(\log \tilde{X}) \simeq \mathcal{O}_{\tilde{U}} \otimes_{\mathbf{Z}} N^{*}$. Hence by the above lemma, we have:

LEMMA 4.3. There exist $g^{Z}$-equivariant isomorphisms $H^{0}\left(\widetilde{U}, \Theta_{\tilde{U}}(-\right.$ $\log \widetilde{X})) \simeq N_{c}$ and $H^{0}\left(\widetilde{U}, \Omega_{\widetilde{U}}^{1}(\log \widetilde{X})\right) \simeq N_{c}^{*}$.

Lemma 4.4. $H^{p}\left(g^{z}, C\right) \simeq C$ for $p=0,1$,

$$
H^{p}\left(g^{z}, N_{c}\right) \simeq\left\{\begin{array}{lll}
\operatorname{ker}(g-1) & \text { for } & p=0 \\
\operatorname{coker}(g-1) & \text { for } & p=1
\end{array}\right.
$$

and

$$
H^{p}\left(g^{z}, N_{c}^{*}\right) \simeq\left\{\begin{array}{lll}
\operatorname{ker}\left({ }^{t} g-1\right) & \text { for } & p=0 \\
\operatorname{coker}\left({ }^{t} g-1\right) & \text { for } & p=1
\end{array} .\right.
$$

Proof. Clearly, $H^{0}\left(g^{z}, N_{c}\right)=\left(N_{c}\right)^{g^{Z}}=\operatorname{ker}(g-1)$ and $H^{0}\left({ }^{t} g^{z}, N_{c}^{*}\right)=$ $\left(N_{c}^{*}\right)^{t_{g} Z}=\operatorname{ker}\left({ }^{t} g-1\right)$. Since $g^{Z}$ (resp. $\left.{ }^{t} g^{Z}\right)$ is generated by $g$ (resp. ${ }^{t} g$ ), we have $Z^{1}\left(g^{Z}, N_{c}\right) \simeq N_{c}$ (resp. $\left.Z^{1}\left({ }^{t} g^{Z}, N_{c}^{*}\right) \simeq N_{c}^{*}\right)$ and $B^{1}\left(g^{Z}, N_{c}\right) \simeq \operatorname{Im}(g-1)$ (resp. $\left.B^{1}\left({ }^{t} g^{z}, N_{c}^{*}\right) \simeq \operatorname{Im}\left({ }^{t} g-1\right)\right)$. Hence $H^{1}\left(g^{z}, N_{c}\right) \simeq \operatorname{coker}(g-1)$ (resp. $\left.H^{1}\left({ }^{t} g^{Z}, N_{c}^{*}\right) \simeq \operatorname{coker}\left({ }^{t} g-1\right)\right)$. Since $g^{Z}$ acts on $H^{0}\left(\widetilde{U}, \mathcal{O}_{\widetilde{U}}\right) \simeq C$ trivially, we get $H^{0}\left(g^{\boldsymbol{Z}}, \boldsymbol{C}\right) \simeq \boldsymbol{C}$ and $H^{1}\left(g^{\boldsymbol{Z}}, \boldsymbol{C}\right)=\operatorname{Hom}\left(g^{\boldsymbol{Z}}, \boldsymbol{C}\right) \simeq \boldsymbol{C}$.

q.e.d.

Next, we show that $E_{2}^{p, q}\left(g^{z}, \tilde{\mathscr{F}}\right)=0$ for $q \geqq 1$. Let $l$ be an integer such that $g^{l}$ and $\Sigma$ satisfy the condition of Theorem 2.2 and let $U^{\prime}=$ $\tilde{U} / g^{l Z}\left(\right.$ resp. $\left.X^{\prime}=\tilde{X} / g^{l Z}\right)$. Then $U^{\prime}$ (resp. $X^{\prime}$ ) is an $l$-sheeted unramified covering of $U$ (resp. $X$ ). By Theorem 2.2, we have a degeneration $\varphi: \mathscr{U} \rightarrow \boldsymbol{P}^{1}$ of $U^{\prime}$ and a divisor $\mathscr{Z}$ on $\mathscr{U}$ such that $\varphi^{-1}(t) \simeq U^{\prime}\left(\varphi^{-1}(t) \cap\right.$ $\mathscr{C} \simeq X^{\prime}$ ) for $t \neq 0, \infty$ and that $U_{0}:=\varphi^{-1}(0)$ is an irreducible variety we obtain by identifying two disjoint divisors of a toric variety. Let $\Theta_{\mathscr{K}}(-\log \mathscr{Q})$ be the subsheaf of the tangent sheaf $\Theta_{\mathscr{K}}$ of $\mathscr{U}$ consisting of germs of holomorphic derivatives $\delta$ with $\delta I \subset I$ and let $\Omega_{\mathscr{Z}}^{1}(\log \mathscr{Z})$ be the dual sheaf of $\Theta_{\mathfrak{U}}(-\log \mathscr{X})$, where $I \subset \mathcal{O}_{\mathfrak{U}}$ is the ideal of definition for $\mathscr{X}$.

LEMma 4.5 .

$$
\begin{aligned}
\operatorname{dim} H^{p}\left(U_{0}, \mathcal{O}_{U_{0}}\right) & =\left\{\begin{array}{lll}
1 & \text { for } & p=0,1 \\
0 & \text { for } & p \geqq 2,
\end{array}\right. \\
\operatorname{dim} H^{p}\left(U_{0}, \Theta_{\mathfrak{x}}(-\log \mathscr{Q})_{0}\right)= & \left\{\begin{array}{lll}
\operatorname{dim} N_{c}^{g l z}+1 & \text { for } & p=0,1 \\
0 & \text { for } & p \geqq 2
\end{array}\right.
\end{aligned}
$$

and

$$
\operatorname{dim} H^{p}\left(U_{0}, \Omega_{\mathscr{Z}}^{1}(\log \mathscr{Z})_{0}\right)=\left\{\begin{array}{lll}
\operatorname{dim}\left(N_{\boldsymbol{c}}^{*}\right)^{t_{g} l \mathbf{Z}}+1 & \text { for } & p=0,1 \\
0 & \text { for } & p \geqq 2
\end{array}\right.
$$


Proof. Let $D$ be the double locus of $U_{0}$ and let $\hat{U}_{0}$ be the normalization of $U_{0}$. Then we have an exact sequence

$$
0 \rightarrow \mathcal{O}_{U_{0}} \rightarrow \mathcal{O}_{\hat{U}_{0}} \rightarrow \mathcal{O}_{D} \rightarrow 0 .
$$

Since $\hat{U}_{0}$ and $D$ are compact toric varieties and since $\mathscr{G} \otimes \mathcal{O}_{\hat{V}_{0}}$ and $\mathscr{G} \otimes \mathcal{O}_{D}$ are free sheaves on $\hat{U}_{0}$ and $D$, respectively, we have $H^{p}\left(\hat{U}_{0}, \mathscr{G} \otimes \mathcal{O}_{\hat{U}_{0}}\right)=$ $H^{p}\left(D, \mathscr{G} \otimes \mathcal{O}_{D}\right)=0$ for $p>0$, where $\mathscr{G}=\mathcal{O}_{k}, \Theta_{k}(-\log \mathscr{Q})$ or $\Omega_{u l}^{1}(\log \mathscr{Z})$. Hence $H^{0}\left(U_{0}, \mathscr{G} \otimes \mathcal{O}_{U_{0}}\right)=\operatorname{ker}(d), H^{1}\left(U_{0}, \mathscr{G} \otimes \mathcal{O}_{U_{0}}\right)=\operatorname{coker}(d)$ and $H^{p}\left(U_{0}, \mathscr{G} \otimes\right.$ $\left.\mathcal{O}_{U_{0}}\right)=0$ for $p \geqq 2$, where $d: H^{0}\left(\hat{U}_{0}, \mathscr{G} \otimes \mathcal{O}_{\hat{U}_{0}}\right) \rightarrow H^{0}\left(D, \mathscr{G} \otimes \mathcal{O}_{D}\right)$. Here $H^{0}\left(\hat{U}_{0}, \mathscr{G} \otimes \mathcal{O}_{\hat{U}_{0}}\right)=H^{0}\left(D, \mathscr{G} \otimes \mathcal{O}_{D}\right)=C, N_{c} \oplus C$ or $N_{c}^{*} \oplus C^{*}$ and $d=0$, $\left(\widehat{g}_{+}\right)^{l}-1\left(=\left(g^{l}-1,0\right)\right)$ or $\left({ }^{t} \hat{g}_{+}\right)^{l}-1 \quad\left(=\left({ }^{t} g^{l}-1,0\right)\right)$, according as $\mathscr{G}=$ $\mathcal{O}_{\mathscr{L}}, \Theta_{u}(-\log \mathscr{Z})$ or $\Omega_{u}^{1}(\log \mathscr{Z})$.

q.e.d.

Since $\varphi^{-1}\left(\boldsymbol{C}^{\times}\right) \simeq U^{\prime} \times \boldsymbol{C}^{\times} \quad\left(\phi^{-1}\left(\boldsymbol{C}^{\times}\right) \cap \mathscr{Q} \simeq X^{\prime} \times \boldsymbol{C}^{\times}\right)$, we see that $\Theta_{\mathscr{R}}(-\log \mathscr{Z})_{t} \simeq \Theta_{U^{\prime}}\left(-\log X^{\prime}\right) \oplus \mathcal{O}_{U^{\prime}}$ and that $\Omega_{u}^{1}(\log \mathscr{Z})_{t} \simeq \Omega_{u}^{1}\left(\log X^{\prime}\right) \oplus \mathcal{O}_{U^{\prime}}$ for each $t \in C^{\times}$. Hence by the upper semi-continuity [1, Theorem 4.12], we have

$\operatorname{dim} H^{p}\left(U^{\prime}, \mathscr{O}_{U^{\prime}}\right) \leqq \operatorname{dim} H^{p}\left(U_{0}, \mathcal{O}_{U_{0}}\right)$, $\operatorname{dim} H^{p}\left(U^{\prime}, \Omega_{U^{\prime}}^{1}\left(\log X^{\prime}\right)\right)+\operatorname{dim} H^{p}\left(U^{\prime}, \mathcal{O}_{U^{\prime}}\right) \leqq \operatorname{dim} H^{p}\left(U_{0}, \Omega^{1}{ }_{u}(\log \mathscr{Q})_{0}\right)$ and

$\operatorname{dim} H^{p}\left(U^{\prime}, \Theta_{U^{\prime}}\left(-\log X^{\prime}\right)\right)+\operatorname{dim} H^{p}\left(U^{\prime}, \mathcal{O}_{U^{\prime}}\right) \leqq \operatorname{dim} H^{p}\left(U_{0}, \Theta_{4}(-\log \mathscr{Z})_{0}\right)$. On the other hand, by Lemma $4.3, \operatorname{dim} H^{p}\left(g^{l z}, F\right)=\operatorname{dim} E_{2}^{p, 0}\left(g^{l z}, \tilde{F}\right) \leqq$ $\operatorname{dim} H^{p}\left(U^{\prime}, \mathscr{F}^{\prime}\right)$, where $F=C, N_{\boldsymbol{c}}$ or $N_{c}^{*}$, and $\mathscr{F}^{\prime}=\mathcal{O}_{U^{\prime}}, \Theta_{U^{\prime}}\left(-\log X^{\prime}\right)$ or $\Omega_{U^{\prime}}^{1}\left(\log X^{\prime}\right)$, according as $\mathscr{F}=\mathcal{O}_{\tilde{U}}, \Theta_{\tilde{U}}(-\log \widetilde{X})$ or $\Omega_{\widetilde{U}}^{1}(\log \widetilde{X})$. Hence by Lemmas 4.3, 4.4 and 4.5 , we obtain the equalities $\operatorname{dim} E_{2}^{p, o}\left(g^{l z}, \tilde{F}\right)=$ $\operatorname{dim} H^{p}\left(U^{\prime}, \mathscr{F}^{\prime}\right)$, because $\operatorname{dim}\left(N_{c}\right)^{g^{l Z}}=\operatorname{dim} \operatorname{ker}\left(g^{l}-1\right)=\operatorname{dim} \operatorname{coker}\left(g^{l}-1\right)$ and $\operatorname{dim}\left(N_{c}^{*}\right)^{t_{g} l z}=\operatorname{dim} \operatorname{ker}\left({ }^{t} g^{l}-1\right)=\operatorname{dim} \operatorname{coker}\left({ }^{t} g^{l}-1\right)$. Therefore, we have $E_{2}^{p, q}\left(g^{l z}, \tilde{F}\right)=0$ for $q \geqq 1$. Then by the Hochschild-Serre exact sequence, we have $E_{2}^{p, q}\left(g^{Z}, \tilde{\mathscr{F}}\right)=H^{p}\left(g^{z}, H^{q}(\widetilde{U}, \tilde{\mathscr{F}})\right)=H^{p}\left(g^{\mathbf{Z}} / g^{l \mathbf{Z}}, H^{q}(\widetilde{U}, \tilde{\mathscr{F}})^{g^{l Z}}\right)=0$ for $q \geqq 1$. Hence $H^{p}(U, \mathscr{F})=E_{2}^{p, 0}\left(g^{z} \tilde{\mathscr{F}}\right)$. Thus we complete the proof of Proposition 4.1 , by Lemmas 4.3 and 4.4 .

PROPOSITION 4.6.

$$
\operatorname{dim} H^{i}\left(U, \Omega_{U}^{1}\right)=\left\{\begin{array}{lll}
0 & \text { for } & i \neq 1 \\
s & \text { for } & i=1
\end{array},\right.
$$

where $s$ is the number of the irreducible components of $X$.

PRoof. Let $\boldsymbol{R}_{\geq 0} n_{1}+\boldsymbol{R}_{\geq 0} n_{2}+\cdots+\boldsymbol{R}_{\geq 0} n_{r}$ be an $r$-dimensional nonsingular cone in $\Sigma$ and let $\left\{m_{1}, m_{2}, \cdots, m_{r}\right\}$ be the $Z$-basis of $N^{*}$ dual to 
$\left\{n_{1}, n_{2}, \cdots, n_{r}\right\}$. Let $\boldsymbol{e}(m)$ be the same as in the proof of Lemma 4.2 and let $\omega_{j}=d \boldsymbol{e}\left(m_{j}\right) / \boldsymbol{e}\left(m_{j}\right)$ for $j=1$ through $r$. Then $\left\{\omega_{1}, \omega_{2}, \cdots, \omega_{r}\right\}$ is a $\boldsymbol{C}$ basis of $H^{\circ}\left(\tilde{U}, \Omega_{\widetilde{U}}^{1}(\log \tilde{X})\right) \simeq N_{c}^{*}$. Here we note that $\omega_{j}$ has poles along $\operatorname{orb}\left(\boldsymbol{R}_{\geq 0} n_{j}\right)$ and does not have poles along $\operatorname{orb}\left(\boldsymbol{R}_{\geq 0} n_{k}\right)$ with $k \neq j$, because $\left\langle m_{j}, n_{k}\right\rangle=\delta_{j k}$. Hence any non-zero element of $H^{\circ}\left(U, \Omega_{U}^{1}(\log X)\right) \simeq$ $H^{0}\left(\widetilde{U}, \Omega_{\widetilde{U}}^{1}(\log \widetilde{X})\right)^{g}$ has poles along $X$. Thus we conclude that $H^{0}\left(U, \Omega_{U}^{1}\right)=$ 0 . Next, consider the long exact sequence of the cohomology groups arising from the short exact sequence

$$
0 \rightarrow \Omega_{U}^{1} \rightarrow \Omega_{U}^{1}(\log X) \rightarrow \bigoplus_{k=1}^{s} \mathcal{O}_{\hat{x}_{k}} \rightarrow 0,
$$

where $\hat{X}_{k}$ are the normalizations of the irreducible components $X_{k}$ of $X=X_{1}+X_{2}+\cdots+X_{s}$. Since each $\hat{X}_{k}$ is a compact toric variety, we have $H^{i}\left(\hat{X}_{k}, \mathcal{O}_{\hat{X}_{k}}\right)=0$ for $i>0$ and $H^{0}\left(\hat{X}_{k}, \mathcal{O}_{\hat{X}_{k}}\right) \simeq \boldsymbol{C}$. Hence by Proposition 4.1, we get $H^{i}\left(U, \Omega_{U}^{1}\right)=0$ for $i>1$ and $\operatorname{dim} H^{1}\left(U, \Omega_{U}^{1}\right)=\operatorname{dim} H^{0}\left(U, \Omega_{U}^{1}\right)-$ $\operatorname{dim} \operatorname{ker}\left({ }^{t} g-1\right)+s+\operatorname{dim} \operatorname{coker}\left({ }^{t} g-1\right)=s$.

q.e.d.

When $r=3$, we can determine the dimensions of $H^{q}\left(U, \Omega_{U}^{p}\right)$ for all $p$ and $q$ by the Serre duality. In particular, $\operatorname{dim} H^{0}\left(U, \Omega_{U}^{1}\right)=\operatorname{dim} H^{3}\left(U, \Omega_{U}^{2}\right)=0$ and $\operatorname{dim} H^{1}(U, \mathcal{O})=\operatorname{dim} H^{2}\left(U, \Omega_{U}^{3}\right)=1$. Since $b_{1}(U)=b_{5}(U)=1$, the maps $E_{1}^{0,1} \rightarrow E_{1}^{1,1}$ and $E_{1}^{2,2} \rightarrow E_{1}^{3,2}$ must be zero-maps and hence the spectral sequence $E_{1}^{p, q}=H^{q}\left(U, \Omega_{U}^{p}\right) \Rightarrow H^{p+q}(U, C)$ degenerates. Thus we have:

THEOREM 4.7. When $r=3, U$ has the following Betti-numbers: $b_{0}(U)=b_{1}(U)=b_{5}(U)=b_{6}(U)=1, b_{2}(U)=b_{4}(U)=s$ and $b_{3}(U)=0$. Hence the Euler-Poincaré characteristic of $U$ is $\chi(U)=2 s$.

5. Deformations. We keep the notation and the assumption in the previous section. Let $\Theta_{U}$ be the tangent sheaf of $U$.

Proposition 5.1. Assume that the dual graph of $X=X_{1}+X_{2}+\cdots+$ $X_{s}$ is a triangulation. Then $H^{i}\left(U, \Theta_{U}\right) \simeq \bigoplus_{k=1}^{s} H^{i}\left(X_{k}, \mathcal{O}_{X_{k}}\left(X_{k}\right)\right)$, for $i \geqq 2$, $H^{\circ}\left(U, \Theta_{U}\right) \simeq \operatorname{ker}(g-1)$ and there exists an exact sequence

$$
0 \rightarrow H^{1}\left(U, \Theta_{U}(-\log X)\right) \rightarrow H^{1}\left(U, \Theta_{U}\right) \rightarrow \bigoplus_{k=1}^{s} H^{1}\left(X_{k}, \mathcal{O}_{X_{k}}\left(X_{k}\right)\right) \rightarrow 0 .
$$

Proof. Consider the long exact sequence of cohomology groups arising from the short exact sequence of sheaves

$$
0 \rightarrow \Theta_{U}(-\log X) \rightarrow \Theta_{U} \rightarrow \bigoplus_{k=1}^{s} \mathcal{O}_{X_{k}}\left(X_{k}\right) \rightarrow 0 .
$$

Then by Proposition 4.1, it is sufficient to show that $H^{0}\left(X_{k}, \mathcal{O}_{X_{k}}\left(X_{k}\right)\right)=0$, for each irreducible component $X_{k}$ of $X$. Let $Y$ be an irreducible component of $\widetilde{X}$ such that the image $q(Y)$ of $Y$ under the quotient map $q: \widetilde{X} \rightarrow X$ is $X_{k}$. Then $Y$ is the closure of the orbit $\operatorname{orb}\left(\boldsymbol{R}_{\geq 0} n\right)$ corresponding to a one-dimensional cone $\boldsymbol{R}_{\geq 0} n$ in $\Sigma$. Let $n_{1}, n_{2}, \cdots$ and $n_{t}$ be the link of $n$ in $\Sigma$, i.e., $\boldsymbol{R}_{\geq 0} n+\boldsymbol{R}_{\geq 0} n_{i}(i=1$ through $t)$ are two-dimensional 
cones in $\Sigma$. Then the closures $Y_{i}$ of the orbits $\operatorname{orb}\left(\boldsymbol{R}_{\geq 0} n_{i}\right)$ are the irreducible components of $\tilde{X}$ with $Y_{i} \cap Y \neq \varnothing$. We easily see that $\operatorname{dim} H^{0}\left(Y, \mathcal{O}_{Y}(Y)\right)=\#\left\{m \in N^{*} \mid\langle m, n\rangle=-1,\left\langle m, n_{i}\right\rangle \geqq 0\right.$ for $\left.1 \leqq i \leqq t\right\}$. Suppose that there exists an element $m$ in $N^{*}$ such that $\langle m, n\rangle=-1$ and that $\left\langle m, n_{i}\right\rangle \geqq 0$. Then the convex hull of $\left\{n, n_{1}, \cdots, n_{t}\right\}$ contains the origin, a contradiction to the fact that $\left\{n, n_{1}, \cdots, n_{t}\right\} \subset|\Sigma| \backslash\{0\} \subset H(g)$. Therefore, $H^{0}\left(X_{k}, \mathcal{O}_{X_{k}}\left(X_{k}\right)\right) \simeq H^{0}\left(Y, \mathcal{O}_{Y}(Y)\right)=0$.

q.e.d.

Since the dimension of each irreducible component $X_{k}$ of $X$ is equal to $r-1$, we get $H^{r}\left(X_{k}, \mathcal{O}_{X_{k}}\left(X_{k}\right)\right)=0$. Hence we have:

CoROLLARY 5.2. $H^{r}\left(U, \Theta_{U}\right)=0$.

COROLlary 5.3. When $r=2$, i.e., $U$ is a hyperbolic Inoue surface or a half Inoue surface, we have $\operatorname{dim} H^{1}\left(U, \Theta_{U}\right)=2 s$, where $s$ is the number of the irreducible components of $X$.

Proof. Note that when $r=2$, any $g$ in $K(N)$ and any $g^{z}$-invariant r.p.p. decomposition $\Sigma$ with $|\Sigma|=(H(g) \backslash L(g)) \cup\{0\}$ satisfy the conditions of Corollary 2.3. Since $g$ has two real eigenvalues both of which are not equal to one, we have $H^{1}\left(U, \Theta_{U}(-\log X)\right) \simeq \operatorname{coker}(g-1)=0$. On the other hand, by the Riemann-Roch Theorem, we have

$\operatorname{dim} H^{1}\left(Y, \mathscr{O}_{Y}(Y)\right)=\operatorname{dim} H^{0}\left(Y, \mathscr{O}_{Y}(Y)\right)-1-\operatorname{deg} \mathscr{O}_{Y}(Y)=-1-Y^{2}$,

for each irreducible component $Y$ of $X$, because $Y$ is a rational curve with $Y^{2}<0$. Hence

$$
\begin{aligned}
\operatorname{dim} H^{1}\left(U, \Theta_{U}\right) & =\sum_{k=1}^{s} \operatorname{dim} H^{1}\left(X_{k}, \mathcal{O}_{X_{k}}\left(X_{k}\right)\right) \\
& =\sum_{k=1}^{s}\left(-1-X_{k}^{2}\right)=-s-X^{2}+2 s=2 s,
\end{aligned}
$$

because $-X^{2}=s$, by Nakamura's duality [7].

q.e.d.

Since $H^{2}\left(U, \Theta_{U}(-\log X)\right)=0$, there exists a universal family $\pi:(\mathscr{U}, \mathscr{Z}) \rightarrow$ $D$ of deformations for the pair $(U, X) \simeq\left(\pi^{-1}(0), \pi^{-1}(0) \cap \mathscr{X}\right)$ over a polydisk $D$, i.e., the Kodaira-Spencer map $\rho: T_{0}(D) \rightarrow H^{1}\left(U, \Theta_{U}(-\log X)\right)$ is bijective. In fact, we can construct such a family as follows. By Proposition 4.1 and Lemma 4.4, we have the canonical isomorphisms $H^{1}\left(U, \Theta_{U}(-\log X)\right) \simeq$ $H^{1}\left(g^{z}, N_{c}\right) \simeq \operatorname{coker}(g-1)$. Here we note that $N_{c}=\operatorname{ker}(g-1) \oplus \operatorname{Im}(g-1)$. Let $\hat{g}^{z}$ be the automorphism group of $T \operatorname{emb}(\Sigma) \times \operatorname{ker}(g-1)$ generated by $\hat{g}:(x, t) \mapsto(e(t) \cdot g x, t)$, where $\boldsymbol{e}: N_{c} \rightarrow T$ is the map induced by $\exp (2 \pi \sqrt{-1}$ ? $): C \rightarrow C^{\times}$. Then $\hat{g}^{\mathbf{Z}}$ preserve the open set $\widetilde{U} \times \operatorname{ker}(g-1)$ and has no fixed point on it. Hence $\mathscr{U}:=(\widetilde{U} \times D) / \hat{g}^{Z}$ is a complex manifold and the natural projection $\mathscr{U} \rightarrow D$ onto $D$ is a proper smooth 
map, for a small enough polydisk $D$ in $\operatorname{ker}(g-1)$.

6. Examples. We give five 3-dimensional examples and show a list of analytic invariants for them. Let $\left\{n_{1}, n_{2}, n_{3}\right\}$ be a $Z$-basis of $Z^{3}$.

EXAMPLE 1. $g n_{1}=2 n_{1}+n_{2}+n_{3}, g n_{2}=n_{1}+n_{2}+n_{3}$ and $g n_{3}=n_{1}+n_{2}+2 n_{3}$. $\Sigma=\left\{\right.$ faces of $g^{l} \sigma_{i} \mid l \in Z, i=1$ through 6$\}$, where

$$
\begin{aligned}
& \sigma_{1}=\boldsymbol{R}_{\geqq 0} n_{1}+\boldsymbol{R}_{\geqq 0} n_{2}+\boldsymbol{R}_{\geqq 0}\left(2 n_{1}+n_{2}+n_{3}\right), \\
& \sigma_{2}=\boldsymbol{R}_{\geqq 0} n_{2}+\boldsymbol{R}_{\geqq 0}\left(n_{1}+n_{2}+n_{3}\right)+\boldsymbol{R}_{\geqq 0}\left(2 n_{1}+n_{2}+n_{3}\right), \\
& \sigma_{3}=\boldsymbol{R}_{\geqq 0} n_{2}+\boldsymbol{R}_{\geqq 0}\left(n_{1}+n_{2}+n_{3}\right)+\boldsymbol{R}_{\geqq 0}\left(n_{1}+n_{2}+2 n_{3}\right), \\
& \sigma_{4}=\boldsymbol{R}_{\geqq 0} n_{2}+\boldsymbol{R}_{\geqq 0} n_{3}+\boldsymbol{R}_{\geqq 0}\left(n_{1}+n_{2}+2 n_{3}\right), \\
& \sigma_{5}=\boldsymbol{R}_{\geqq 0} n_{1}+\boldsymbol{R}_{\geqq 0} n_{3}+\boldsymbol{R}_{\geqq 0}\left(n_{1}+n_{2}+2 n_{3}\right) \text { and } \\
& \sigma_{8}=\boldsymbol{R}_{\geqq 0} n_{1}+\boldsymbol{R}_{\geqq 0}\left(2 n_{1}+n_{2}+n_{3}\right)+\boldsymbol{R}_{\geqq 0}\left(n_{1}+n_{2}+2 n_{3}\right) .
\end{aligned}
$$

(See Figure 1.)

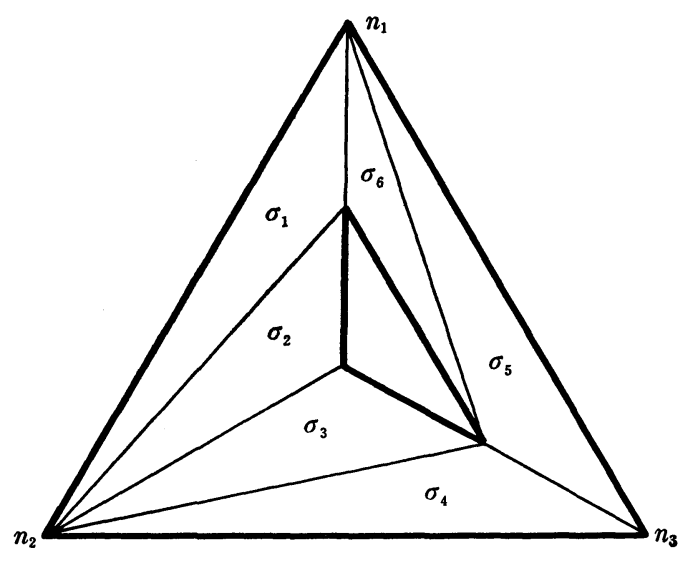

FJGURe 1

EXAMPLE 2. $g n_{1}=2 n_{1}+n_{2}+n_{3}, g n_{2}=n_{1}+n_{2}+2 n_{3}$ and $g n_{3}=n_{1}+n_{2}+n_{3}$. $\Sigma=\left\{\right.$ faces of $g^{l} \sigma_{i} \mid l \in Z, i=1$ through 6$\}$, where $\sigma_{i}$ are the same as in Example 1.

EXAMPLE 3. $g n_{1}=n_{2}, g n_{2}=n_{1}+n_{3}$ and $g n_{3}=n_{1} . \quad \Sigma=$ f faces of $g^{l} \tau_{1}$ and $g^{l} \tau_{2} \mid l \in Z$, where $\tau_{1}=\boldsymbol{R}_{\geq 0} n_{3}+\boldsymbol{R}_{\geq 0}\left(n_{1}+n_{3}\right)+\boldsymbol{R}_{\geq 0}\left(n_{1}+n_{2}+n_{3}\right)$ and $\tau_{2}=\boldsymbol{R}_{\geqq 0} n_{2}+\boldsymbol{R}_{\geqq 0} n_{3}+\boldsymbol{R}_{\geqq 0}\left(n_{1}+n_{2}+n_{3}\right)$. (See Figure 2.)

EXAMPLE 4. $g n_{1}=n_{1}+n_{3}, g n_{2}=n_{1}$ and $g n_{3}=n_{2} . \quad \Sigma=\left\{\right.$ faces of $g^{l} \tau_{1}$ and $\left.g^{l} \tau_{2} \mid l \in Z\right\}$, where $\tau_{1}$ and $\tau_{2}$ are the same as in Example 3 .

EXAMPLE 5. $g n_{1}=n_{1}+n_{2}+n_{3}, g n_{2}=n_{3}$ and $g n_{3}=n_{1} . \quad \Sigma=$ ffaces of $g^{l} \mu_{1}$ and $\left.g^{l} \mu_{2} \mid l \in Z\right\}$, where $\mu_{1}=\boldsymbol{R}_{\geqq 0} n_{1}+\boldsymbol{R}_{\geqq 0} n_{2}+\boldsymbol{R}_{\geqq 0}\left(n_{1}+n_{2}+n_{3}\right)$ and $\mu_{2}=$ 


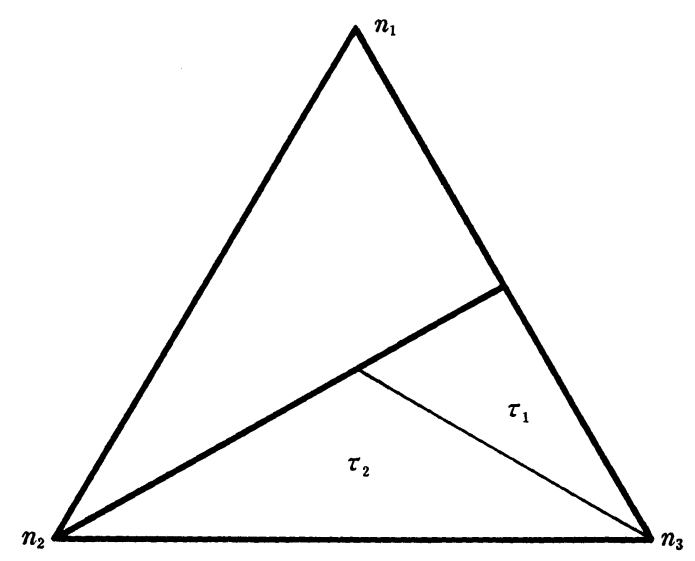

FIGURE 2

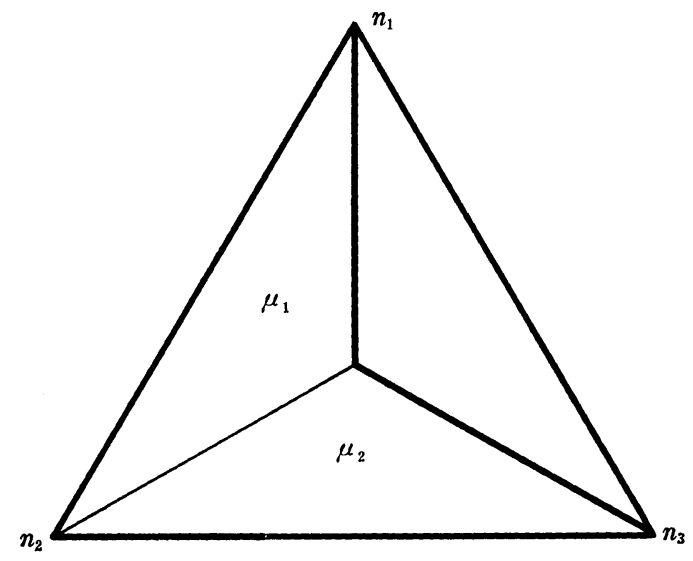

FigURe 3

$\boldsymbol{R}_{\geq 0} n_{2}+\boldsymbol{R}_{\geq 0} n_{3}+\boldsymbol{R}_{\geq 0}\left(n_{1}+n_{2}+n_{3}\right)$. (See Figure 3.)

We easily see that all the above examples satisfy the condition of Definition 1.1. The complex manifolds we obtain from $g$ and $\Sigma$ in Example 1 and Example 2 contain global spherical shells by Theorem 3.2. The complex manifold we obtain from $g$ and $\Sigma$ in Example 4 is bimeromorphic to that in [6]. Although our examples do not satisfy the assumptions of Theorem 5.1, we can calculate the dimensions $h^{i}\left(U, \Theta_{U}\right)$ of $H^{i}\left(U, \Theta_{U}\right)$ as follows. There are positive integers $l$ such that the dual graphs of $X^{\prime}:=\tilde{X} / g^{l z}$ are triangulations. Then $U$ and $X$ are quotients of $U^{\prime}:=\tilde{U} / g^{l z}$ and $X^{\prime}$, respectively, by the finite cyclic groups $G=g^{z} / g^{i z}$, which have no fixed points on $U^{\prime}$. Hence $\Theta_{U} / \Theta_{U}(-\log X)$ are the subsheaves of $q_{*}\left(\Theta_{U^{\prime}} / \Theta_{U^{\prime}}\left(-\log X^{\prime}\right)\right) \simeq q_{*}\left(\bigoplus_{k=1}^{s l} \bigcirc_{X_{k}^{\prime}}\left(X_{k}^{\prime}\right)\right)$ consisting of germs of $G$-invariant 
sections, where $X^{\prime}=X_{1}^{\prime}+X_{2}^{\prime}+\cdots+X_{s l}^{\prime}$ and $q: X^{\prime} \rightarrow X$ is the quotient map. Therefore, $\operatorname{dim} H^{i}\left(U, \Theta_{U} / \Theta_{U}(-\log X)\right)=\operatorname{dim}\left(\bigoplus_{k=1}^{s l} H^{i}\left(X_{k}^{\prime}, \bigcirc_{X_{k}^{\prime}}\left(X_{k}^{\prime}\right)\right)\right)^{a}=$ $(1 / l) \sum_{k=1}^{s l} \operatorname{dim} H^{i}\left(X_{k}^{\prime}, \mathcal{O}_{X_{k}^{\prime}}\left(X_{k}^{\prime}\right)\right)$. Then by Proposition 4.1, Theorem 5.1 and its proof, we have $h^{0}\left(U, \Theta_{U}\right)=\operatorname{dim} \operatorname{ker}(g-1), h^{1}\left(U, \Theta_{U}\right)=\operatorname{dim} \operatorname{coker}(g-1)+$ $(1 / l) \sum_{k=1}^{s l} \operatorname{dim} H^{1}\left(X_{k}^{\prime}, \mathcal{O}_{X_{k}^{\prime}}\left(X_{k}^{\prime}\right)\right)$ and $h_{i}\left(U, \Theta_{U}\right)=(1 / l) \sum_{k=1}^{s l} \operatorname{dim} H^{i}\left(X_{k}^{\prime}, \mathcal{O}_{X_{k}^{\prime}}\left(X_{k}^{\prime}\right)\right)$, for $i \geqq 2$.

\begin{tabular}{l|c|c|c|c|c} 
& $h^{0}(U, \Theta)$ & $h^{1}(U, \Theta)$ & $h^{2}(U, \Theta)$ & $C_{3}=\chi(U)$ & $C_{1}^{3}=X^{3}$ \\
\hline Example 1 & 1 & 8 & 1 & 6 & -18 \\
Example 2 & 0 & 7 & 1 & 6 & -18 \\
Example 3 & 0 & 0 & 0 & 2 & -2 \\
Example 4 & 0 & 1 & 0 & 2 & -4 \\
Example 5 & 0 & 3 & 0 & 2 & -8
\end{tabular}

\section{REFERENCES}

[1] C. BĂNICA AND O. STǍNǍșiLǍ, Algebraic methods in the global theory of complex spaces, Editula Academiei, București and John Wiley \& Sons, London, New York, Sydney and Tronto, 1976.

[2] A. GrothendieCK, Sur quelques points d'algèbre homologique, Tôhoku Math. J. 9 (1957), 119-227.

[3] M. Inout, New surfaces with no meromorphic functions, II, in Complex Analysis and Algebraic Geometry, Iwanami Shoten Publ. and Cambridge Univ. Press, 1977, 91-106,

[4] M.-N. IshidA AND T. ODA, Torus embeddings and tangent complexes, Tôhoku Math. J. 33 (1981), 337-381.

[5] Ma. Kato, Compact complex manifolds containing global spherical shells, I, Proc. Int. Symp. Algebraic Geometry, Kyoto, 1977, Kinokuniya, Tokyo and North-Holland, Amsterdam, New York, Oxford, 45-84.

[6] Ma. Kato, An example of a 3-dimensional complex manifold, Symposium on Algebraic Geometry (In Japanese), Sendai, 1980, 179-190.

[7] I. NakamuRA, Inoue-Hirzebruch surfaces and a duality of hyperbolic unimodular singularities, I, Math. Ann. 252 (1980), 221-235.

[8] T. ODA, Lectures on Torus Embeddings and Applications (Based on joint work with K. Miyake), Tata. Inst. of Fund. Res., Bombay, No. 58, Springer-Verlag, BerlinHeiderberg-New York, 1978.

[9] G. K. Sankaran, Higher-dimensional anlogues of Inoue-Hirzebruch surfaces, Math. Ann. 276 (1987), 515-528.

[10] N. Dunford and J. T. Schwartz, Linear operations Part II, Pure and applied mathematics volume VII, Interscience.

[11] H. SUminiro, Equivariant completion, I, J. Math. Kyoto Univ. 14 (1974), 1-28.

Department of General Education

TôHOKU GAKUIN UNIVERSITY

SENDAI, 980

JAPAN 